\title{
Simulation Based on Trivariate NURBS and Isogeometric Analysis of a Spur Gear
}

\author{
O. T. Yusuf, ${ }^{a}$ Z. Gang, ${ }^{a}$ W. Wei, ${ }^{a}$ and S. O. Onuh ${ }^{\mathrm{b}}$
}

a Mechanical Engineering and Automation, BUAA, Beijing, China

${ }^{\mathrm{b}}$ Center for Satellite Technology Development, NASRDA, Abuja, Nigeria

The approach provides simulation of the gear design with high-precision profile configuration parameters to accurately predict its load capacity and stress state, which requires incessant efforts, as gear failure modes depend largely on its profile representation. The finite element analysis (FEA) is a well-known effective computational method, which many authors used to estimate the gear tooth strength against the primary failure modes of gears. This method is not only known to be time-consuming but often expensive, it employs approximate geometry that resulted in a coarse mesh. The tooth strength and deformation modes were obtained from a three-dimensional gear model based on the trivariate nonuniform rational B-splines (NURBS) under loading and investigated using the isogeometric analysis (IGA) for simulation. The results produced with the IGA simulation are compared with the corresponding values derived from the classical estimation method with the Lewis equation and FEM analysis using ANSYS. Both results are in good agreement and confirm that the IGA model is more accurate than the FEM one. This study demonstrated that IGA was practically applicable, providing an alternative means of using high-precision geometry of $3 D$ models to estimate the behavior of gear bodies under loading and modify their strength calculations.

Keywords: three-dimensional model, carbon steel, spur gear, gear profile, trivariate NURBS, finite element method, isogeometric analysis.

Introduction. Gears are used in various types of machinery in many engineering areas, including automobiles, marines, aeronautics and astronautics, where extreme operating speeds of rotating members increase the load input on machinery, thereby demanding high strength gearing component to achieve positive power transmission. In operation, the teeth deform elastically under the induced radial and tangential components of pressure load via the contact points on the mating teeth profiles (as shown in Fig. 1) in the direction of the pressure line.

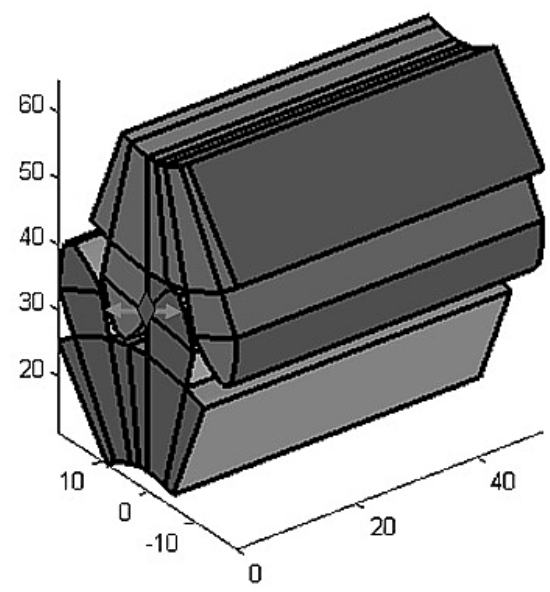

Fig. 1. Loaded solid spur gear contact teeth at the pitch point (diamonds) and transfer along the pressure line (arrows). 
Fatigue failure of gear tooth occur, as a result of high stress concentration at the root of a gear tooth subjected to bending loads. To achieve high-strength gearing with the highest efficiency power transmission requires a design minimizing this bending stress. Ristić [1] investigated the optimal fillet tooth root radius to minimize the root stress intensity in order to create a valid numerical model for a tooth root geometrical discontinuity at static loading using finite element method (FEM). As shown in study [2], modification of root fillet profile using a circular root fillet instead of the standard trochoidal one can improve the tooth strength in a spur gear. These investigations bordered on accuracy and geometrical modification of gear profile that can be easily achieved using the volumetric polynomial functions, such as tensor product NURBS [3], which allows geometries to be represented exactly with a smooth surface that can be directly used in the IGA [4]. IGA is a way of invoking the isoparametric concept of representing the field variables with the same smooth NURBS basis functions for numerical computation. Moreover, IGA allows for an easy model modification. With NURBS model, one can exclude geometric discontinuities induced by the conventional geometry discretization (which causes jumps in the contact surfaces unless finest meshes are used), as well as avoid the time- and memory-consuming mesh generation operation for the transformation of CAD model into a finite element (FE) mesh before it can be used in the analysis.

Both theoretical calculation method via the Lewis equation [5], and finite element method have been widely used in the field of gear strength numerical calculation. The solution of load distribution in the meshed gear tooth pairs with numerical FEM [6-8] resulted in many important conclusions on the spur gear stress distrubution.

IGA has been successfully applied in various fields for solving PDEs of domains formed by smooth basis functions of NURBS. Such fields include structural mechanics and optimization [9, 10], solid mechanics [11, 12], fluid mechanics [13], fracture mechanics $[14,15]$, biomechanics [16], and contact mechanics [17-19]. However, this approach has not been fully implemented into commercialized sofware packages due to trimless nature of NURBS for representing complex NURBS domains.

Analysis of gear stress using NURBS-based isogeometric analysis is recently presented for a two-dimensional case [20], but [21] shows that 2D representation method cannot embrace the actual stress field in a spur tooth fillet. We generalize this idea to create an accurate $3 \mathrm{D}$ conforming multi-patch gear tooth model based on NURBS and investigate the stress-strain states and deflection of the gear under the influence of loads at the points of contact using the isogeometric analysis. This provides more insight into the load distribution along the tooth face width of gear, as well as stress distribution in the gear tooth root. The numerical results fobtained by IGA are compared to those derived from the available empirical and finite element analyses from ANSYS workbench 14.0 and are found to be in a good agreement.

\section{Basic Characteristics of the Analyzed Spur Gear Model.}

1.1. Gear Geometrical Data. The gear geometrical parameters used in this study are shown in Table 1. These parameters are used to develop a pair model of solid spur gears in contact (as shown in Fig. 1) to illustrate the active gear contact zone, where the maximum torque is transferred along the line of action between the mating gears.

1.2. Materials of Spur Gear. In the manufacturing of spur gear, wide varieties of materials are used, including steels, nylon, aluminum, bronze, cast iron, phenolic, bakelite, and plastics. In this study, a steel gear made from $20 \%$ carbon steel with no subsequent treatment with the properties shown in Table 2 is used.

1.3. Trivariate NURBS. NURBS solids $S(\xi, \eta, \zeta)$ have a topological structural form with grids of control points $P_{i j k}^{w} \in R^{4}$ with associated weights $\left\{w_{i j k}\right\}_{i j k=1}^{n m l}$ that provide the basic information on their geometry, such as position, size, and aspect ratios of the isogeometric mesh. The node vectors in parametric directions $\xi, \eta$, and $\xi$, partition the 
$\mathrm{T}$ a b 1 e 1

\section{Spur Gear Geometrical Data}

\begin{tabular}{||l|c|c|c||}
\hline \multicolumn{1}{|c|}{ Description } & Symbol & Formula & Values \\
\hline Number of teeth & $z$ & - & 12 \\
\hline Pressure angle (deg) & $\theta$ & - & 14.5 \\
\hline Diametral pitch & $P_{d}$ & $h_{a}=0.3183 p$ & 4 \\
\hline Addendum (mm) & $h_{a}$ & $D_{b}=d \cos \theta$ & 6.35 \\
\hline Base circle diameter (mm) & $D_{b}$ & $\pi m$ & 73.8 \\
\hline Circular pitch (mm) & $p$ & $p / 2$ & 19.95 \\
\hline Circular tooth thickness (mm) & $c t t$ & $1.25 m$ & 9.97 \\
\hline Dedendum (mm) & $h_{f}$ & $d / z$ & 7.94 \\
\hline Module (mm) & $m$ & $m(z+2)$ & 6.35 \\
\hline Outside diameter (mm) & $D_{0}$ & $z m$ & 88.9 \\
\hline Pitch circle diameter (mm) & $d$ & - & 76.2 \\
\hline Face width (mm) & $F$ & $2.25 m$ & 50.8 \\
\hline Whole depth (mm) & $h$ & $0.25 m$ & 14.28 \\
\hline Top land width (mm) & $t_{0}$ & & 1.59 \\
\hline
\end{tabular}

$\mathrm{T}$ a b 1 e 2

Properties of Material

\begin{tabular}{|c|c|c|c||}
\hline Properties & Gear & Properties & Gear \\
\hline Material & Carbon steel & Poisson's ratio & 0.3 \\
\hline Elastic modulus $(\mathrm{GPa})$ & 210 & Density $\left(\mathrm{kg} / \mathrm{m}^{3}\right)$ & 7850 \\
\hline Yield strength $(\mathrm{MPa})$ & 758 & & \\
\hline
\end{tabular}

parametric domain of the basis functions $N_{i, p}(\xi), M_{j, q}(\eta)$, and $L_{k, r}(\zeta)$ into piecewise rational polynomial functions $R_{i j k}^{p q r}(\xi, \eta, \zeta)$.

The result of mapping of the parametric domain $\hat{\Omega}$ into the physical domain $\Omega$ is given as $S: \hat{\Omega} \rightarrow \Omega$ with

$$
\begin{gathered}
S^{w}(\xi, \eta, \zeta)=\sum_{i=1}^{n} \sum_{j=1}^{m} \sum_{k=1}^{1} N_{i, p}(\xi) M_{j, q}(\eta) L_{k, r}(\zeta) P_{i j k}^{w}= \\
=\sum_{i=1}^{n} \sum_{j=1}^{m} \sum_{k=1}^{1} w_{i j k} N_{i, p}(\xi) M_{j, q}(\eta) L_{k, r}(\zeta) P_{i j k}
\end{gathered}
$$

in the perspective division of homogenous coordinates:

$$
W(\xi, \eta, \zeta)=\sum_{i=1}^{n} \sum_{j=1}^{m} \sum_{k=1}^{1} N_{i, p}(\xi) M_{j, q}(\eta) L_{k, r}(\zeta) w_{i j k} .
$$


NURBS solid $\in R^{3}$ can be realized using

$$
\begin{gathered}
S(\xi, \eta, \zeta)=\frac{1}{W(\xi, \eta, \zeta)} \sum_{i=1}^{n} \sum_{j=1}^{m} \sum_{k=1}^{1} N_{i, p}(\xi) M_{j, q}(\eta) L_{k, r}(\zeta) w_{i j k} P_{i j k}= \\
=\sum_{i=1}^{n} \sum_{j=1}^{m} \sum_{k=1}^{1} R_{i j k}^{p q r}(\xi, \eta, \zeta) P_{i j k} .
\end{gathered}
$$

Figure 2 shows an example of a quadratic model of trivariate NURBS constructed from uniform open node vectors $\xi=\{0,0,0,1,1,1\}, \eta=\{0,0,0,1,1,1\}$, and $\xi=\{0,0,0,1,1,1\}$. Its associated control grid, as shown in Fig. $2 \mathrm{~b}$, is constructed from piecewise interpolation of 27 control points. Figure $2 \mathrm{c}$ shows the result of the nodal spans for a hexahedron element achieved by mapping of a parametric domain into a physical one via Eqs. (1)-(3).
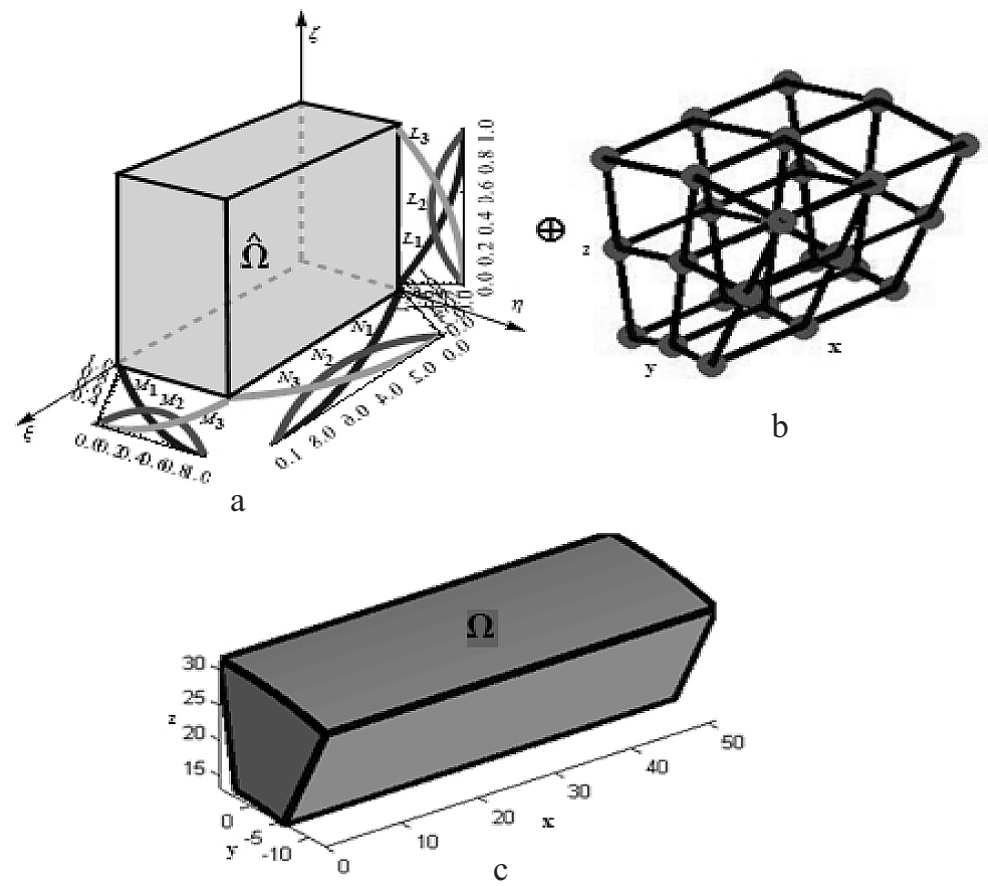

Fig. 2. Parametric domain with basis functions (a); physical domain (b); trivariate NURBS model (c).

2. Modeling of NURBS Based Spur Gear. The specified primary parameters in the previous section serve as input to define the topological part of solids NURBS $S(\xi, \eta, \zeta)$ geometry, in which the gear coordinate data (control points) $\left\{P_{i j k}\right\}_{i j k=1}^{n m l}$, associated with weights $\left\{w_{i j k}\right\}_{i j k=1}^{n m l}$, are embedded into four-dimensional homogeneous coordinates in the projective space $R^{4}$. To obtain the spur gear generation, each tooth of the gear of twelve teeth, in view of their symmetry, comprises of four conformal NURBS patches with three coupling interfaces defined by the product of node vectors of non-decreasing positive real numbers in $\xi, \eta$, and $\xi$ parametric directions given as $\xi=\{0,0,0,1,1,1\}, \quad \eta=$ $=\{0,0,0,1,1,1\}$, and $\zeta=\{0,0,0,1,1,1\}$, from which the basis functions $\left\{N_{i j k}^{p q r}(\xi, \eta, \zeta\}_{i j k=1}^{m n l}\right.$ of degree $p=2, q=2$, and $r=2$ are defined. The association of the 
control points with the basis functions defines one patch of the tooth solid NURBS using Eqs. (1)-(3), this procedure is repeated for other three patches to obtain one-twelve of a single tooth sector spur gear shown in Fig. 3 with the four patches showing in different colors.

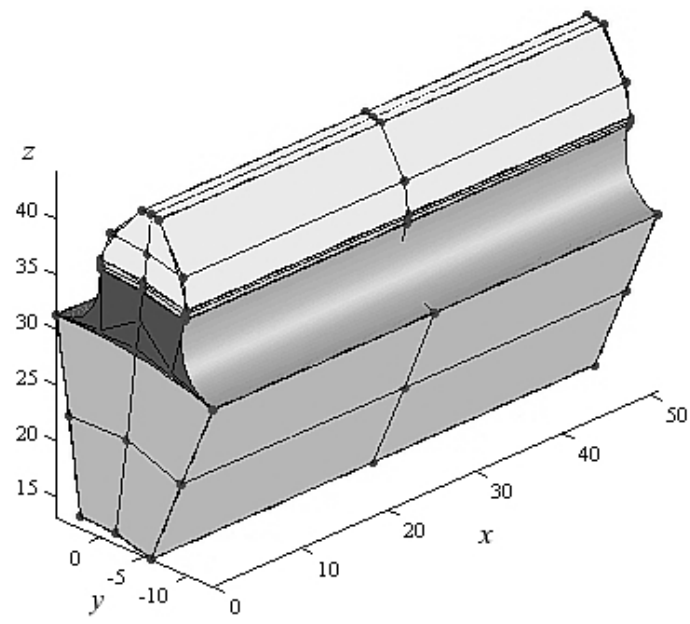

Fig. 3

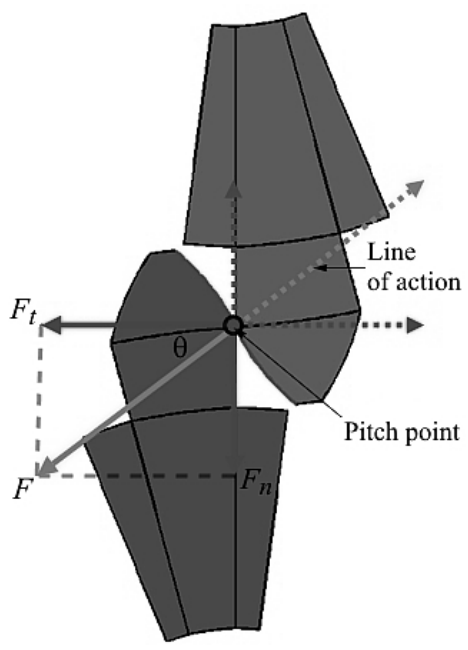

Fig. 4

Fig. 3. Geometrical NURBS model of a single-tooth solid gear.

Fig. 4. Direction of forces acting on the mating gear teeth.

2.1. Theoretical Determination of Gear Load Capacity and Tooth Bending Stress. The spur gear strength is expressed in terms of the bending strength that can be estimated with the Lewis equation [5], which is related to the power and forces to be transmitted via the line of action between the pinion and gear. As illustrated in Fig. 4, the maximum transmission force $F$, which is between the contacting teeth at pressure angle of $\theta$ to pitch line tangent, can be resolved into a tangential component $F_{t}$ and a separating component $F_{n}$. We first compute the fundamental equation relating the tangential force $F_{t}$ at the pitch circle, power $P$ and torque $T$ to have:

$$
\begin{gathered}
F_{t}=\frac{102 P}{v}=\frac{1.95 \cdot 10^{6} P}{d_{w} n}=\frac{2000 T}{d_{w}}, \\
P=\frac{F_{t} v}{102}=\frac{10^{-6}}{1.95} F_{t} d_{w} n, \\
T=\frac{F_{t} d_{w}}{2000}=\frac{974 P}{n}, \\
F_{n}=F_{t} \tan \theta, \\
F=\frac{F_{t}}{\cos \theta}, \\
v=\frac{d_{w} n}{19100},
\end{gathered}
$$


where $v$ is the tangential speed of working pitch circle $\left(\mathrm{ms}^{-1}\right), d_{w}$ is working pitch diameter, $d_{w}=76.2 \mathrm{~mm}, n$ is rotating speed, $n=1000 \mathrm{rpm}$,

$$
\begin{gathered}
v=\frac{d_{w} n}{19100}=\frac{76.2 \cdot 1000}{19100}=3.99 \mathrm{~ms}^{-1}, \\
T=\frac{F_{t} d_{w}}{2000}=\frac{974 P}{n}=\frac{974 \cdot 5}{1000}=4.87 \mathrm{kgf} \cdot \mathrm{m}=47.76 \mathrm{~N} \cdot \mathrm{m}, \\
F_{t}=\frac{102 P}{v}=\frac{1.95 \cdot 10^{6} P}{d_{w} n}=\frac{2000 T}{d_{w}}=\frac{2000 \cdot 4.87}{76.2}=127.82 \mathrm{kgf}=1253.5 \mathrm{~N}, \\
F_{n}=F_{t} \tan \theta=1253.5 \cdot 0.2586=324.16 \mathrm{~N} .
\end{gathered}
$$

The Barth velocity factor $K_{v}$ increases the Lewis stress approximately by

$$
K_{v}=\frac{6.1+V}{6.1}
$$

where $V$ is the velocity at the pitch diameter (in $\mathrm{m} / \mathrm{s}$ ),

$$
K_{v}=\frac{6.1+3.989}{6.1}=1.65
$$

The combined expression for tooth bending stress $\sigma_{b}$ is then

$$
\sigma_{b}=\frac{K_{v} F_{t}}{b_{a} m Y}
$$

where $b_{a}$ is face width (mm), $m$ is module $(\mathrm{mm})$, and $Y$ is the Lewis form factor,

$$
\sigma_{b}=\frac{1.65 \cdot 1253.5}{50.8 \cdot 6.35 \cdot 0.355}=18.06 \mathrm{MPa}, \quad \sigma_{\text {theor }}=\sigma_{b}=18.06 \mathrm{MPa} .
$$

2.2. Construction of a Gear Tooth Fillet. The gear root fillet radius $\left(r_{f}\right)$ is the critical cross section of a gear tooth root as it is exposed to bending and combined action of the radial and tangential components of pressure load, which has a direct influence on the stress concentration factor and thus affects the gear service life. The fillet does not have a constant radius, but is shaped as a curve generated by a hobbing tool or cutter [22] with a tip radius $\left(r_{t}\right)$ for $14.5^{\circ}$ pressure angle which is standardized as

$$
r_{t}=\frac{0.235}{P_{d}},
$$

where $P_{d}$ is diametral pitch, $P_{d}=4, r_{t}=0.235 / P_{d}=0.235 / 4=0.05875$ in $\approx 1.49 \mathrm{~mm}$.

The radius of a gear tooth fillet produced by the tool of radius $r_{t}$ is denoted by

$$
r_{f}=\frac{\left(h_{f}-r_{t}\right)^{2}}{Z /\left(2 P_{d}\right)+\left(h_{f}-r_{t}\right)}+r_{t},
$$




$$
r_{f}=\frac{(7.94-1.49)^{2}}{12 / 8+7.94-1.49}+1.49=6.72 \mathrm{~mm}
$$

\section{Finite Element Analysis Using ANSYS.}

3.1. Models and Mesh. Due to the symmetry of the gear structure and loading, only one-twelfth of the spur gear was modeled using Rhinoceros V5 software as shown in Fig. 5. In its IGES format, it was exported to the commercial finite element package ANSYS workbench 14.0, which was used for a linear static finite element analysis. Three-dimensional isoperimetric hexahedron solid elements defined by eight nodes were used for generating the FEA mesh. For improvement of the calculation accuracy, a fine mesh of hexahedron solid elements is used consisting of 19,737 elements and 86,722 nodes.

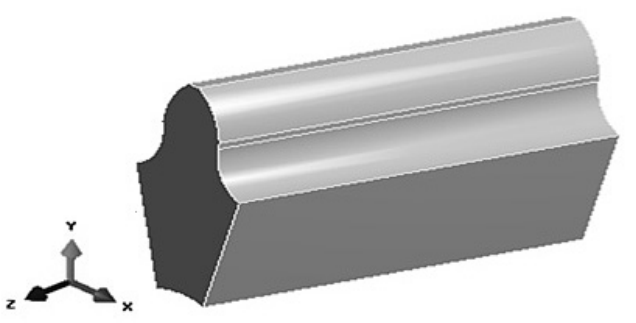

Fig. 5. 3D gear tooth CAD model.

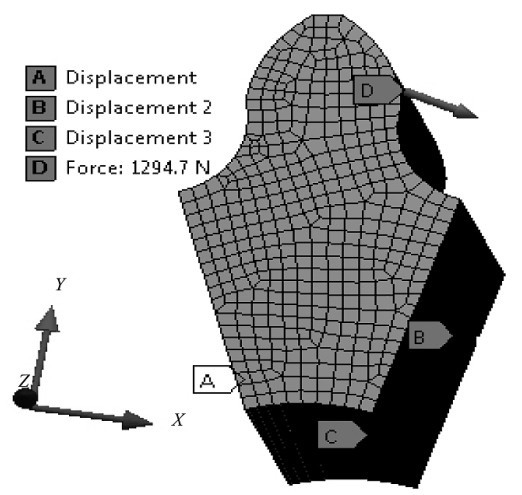

Fig. 6. 3D FEM boundary and loading conditions.

3.2. Boundary and Loading Conditions. The boundary conditions implies the application of displacement constraints and external loads on the 3D FEM mesh. All nodes in the symmetric sections are constrained against displacement in the direction perpendicular to the symmetry plane, i.e., along the surfaces that separate the modeled gear segment from the rest of the gear body. Displacement constraints of fixed support are also placed at the nodes on gear tooth of the inner bore border. The FEA models were subjected to the combined load by the tangential force of $1253.5 \mathrm{~N}$ and the normal force of $324.16 \mathrm{~N}$ at the pressure angle to pitch line tangent. Figure 6 illustrates the finite element mesh boundary and loading conditions for the single-tooth model.

3.3. Material Properties. The material parameters applied in the model include the Young modulus and Poisson's ratio for the carbon steel as specified in Table 2.

4. Isogeometric Analysis of Spur Gear. The IGA simulation, which is an alternative to the traditional finite elements, is based the geometric input model composed by four NURBS patches as shown in Fig. 7 (level 4) with three coupling interfaces. The model is discretized using the tricubic functions (for patch 1, patch 2, patch 3, and patch 4), each consisting of $16 \times 16 \times 16$ elements. This results in the total number of 16,384 elements and 19,652 control points.

4.1. Mesh Definition and Model Refinement. The physical domain $\Omega \in R^{3}$ of the spur gear tooth is represented by four single-patch NURBS mappings $S^{(i)}$ for $i=1$ to 4 , each of which maps the parameter domain $\hat{\Omega}$ into the patch $\Omega^{(i)}=S^{(i)}(\hat{\Omega})$. The superscript $i$ represents the node vectors, degrees, NURBS basis functions, control points associated with the mapping $S^{(i)}$ of the individual patch.

The mesh $M^{(i)}$ is directly defined by the NURBS mapping process of Eqs. (1)-(3), which is quasi-uniform for all $i=1,2,3,4$. The domain is subdivided into subdomains 


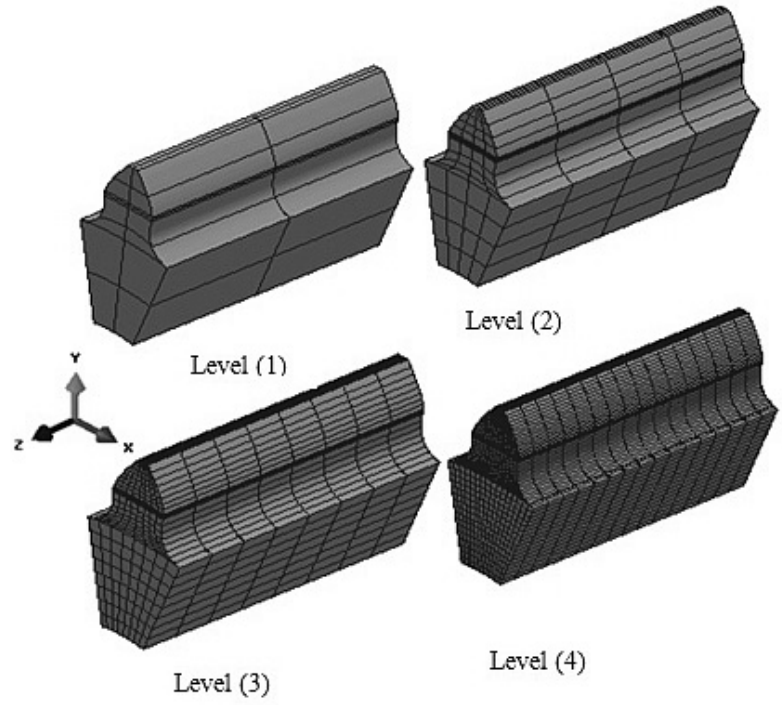

Fig. 7. Four-level refinement of the IGA meshes generated by insertion of nodes.

$\Omega \cup_{e} \Omega^{e}$ by hexahedral elements, such that $\Omega_{i} \cup \Omega_{j} \cup \Omega_{m}=\varnothing$ for $i \neq j \neq m$. The elements are determined by the nodal spans: $\Omega^{e}=\left[\xi_{i}, \xi_{i+1}\right] \otimes\left[\eta_{j}, \eta_{j+1}\right] \otimes\left[\xi_{m}, \zeta_{m+1}\right]$, where $h_{i}$ denotes the element size of $M^{(i)}$. This yields a family of meshes $\left\{M_{h_{i}}^{(i)}\right\}_{h_{i}}$, such that there exists the most coarse mesh $M_{h_{i 0}}^{(i)}$ that does not provide a sufficiently smooth contour plot, which could be directly used to produce accurate results of the analysis. Four levels of this mesh refinement are executed (as shown in Fig. 7) to have a higher resolution mesh. The latter is suitable to obtain accurate results, and the description of the geometry is fixed at the level of $M_{h_{i 0}}^{(i)}$. When the mesh is refined, the control points are adjusted in a such way that the originality of geometrical shape of the model $S^{(i)}$ remains unchanged. Thus, the geometry and its parameterization are held fixed in the refinement process [23].

The meshing operation in FEA is thereby replaced with the model refinement by simple insertion of nodes into IGA, which results in additional degrees of freedom.

4.2. Performing the IGA Analysis. The boundary conditions for the IGA analysis are similar to those for the FEM analysis (Fig. 6). The loads and material properties for the numerical simulation using IGA are identical to those defined in Subsections 3.2 and 3.3.

An algorithm in matlab codes is developed, adopting some built-in functions and structures within the GeoPDEs, IGA, FEM, and Abaqus NURBS libraries [24-26] to construct the geometry based on the gear model data input, which includes the control points, node vectors, orders, the boundaries and interfaces of the NURBS patches needed to perform the analysis. The topological entities in the model were selected, where force and displacement constraints were set, and performed the analysis using the Abaqus NURBS plugins [26].

5. Results and Discussion. In accordance with theoretical investigations given in [22] and FEM [7, 8], the expected maximum concentrated stress occurring at the fillet root at the base of the gear tooth which is equal to tensile stress that causes crack failure shown in Fig. 8 illustrates distribution of the Mises stresses in the tooth root. Figure 9 shows the corresponding tooth deflections calculated for a single-tooth 3D spur gear geometry based on the NURBS four patches and on the 3D FEM model. The compared values are the equivalent Mises stresses and the total deformation. 


\section{$\mathrm{T}$ a b 1 e 3 \\ Comparison of the Maximum Mises Stresses and the Total Deformed Shape Obtained from IGA and FEA}

\begin{tabular}{||c|c|c|c|c|c|c||}
\hline $\begin{array}{c}\sigma_{\text {theor }}, \\
\mathrm{MPa}\end{array}$ & $\begin{array}{c}\sigma_{F E A}, \\
\mathrm{MPa}\end{array}$ & $\begin{array}{c}\sigma_{I G A}, \\
\mathrm{MPa}\end{array}$ & $\begin{array}{c}\text { Deformation } \\
\text { in FEA }(\mathrm{mm})\end{array}$ & $\begin{array}{c}\text { Deformation } \\
\text { in IGA }(\mathrm{mm})\end{array}$ & $\begin{array}{c}\text { Difference (\%) } \\
\left(\sigma_{\text {theor }}-\sigma_{F E A}\right)\end{array}$ & $\begin{array}{c}\text { Difference }(\%) \\
\left(\sigma_{\text {theor }}-\sigma_{I G A}\right)\end{array}$ \\
\hline 18.06 & 16.89 & 18.45 & 0.0018 & 0.0020 & 6.47 & 2.16 \\
\hline
\end{tabular}
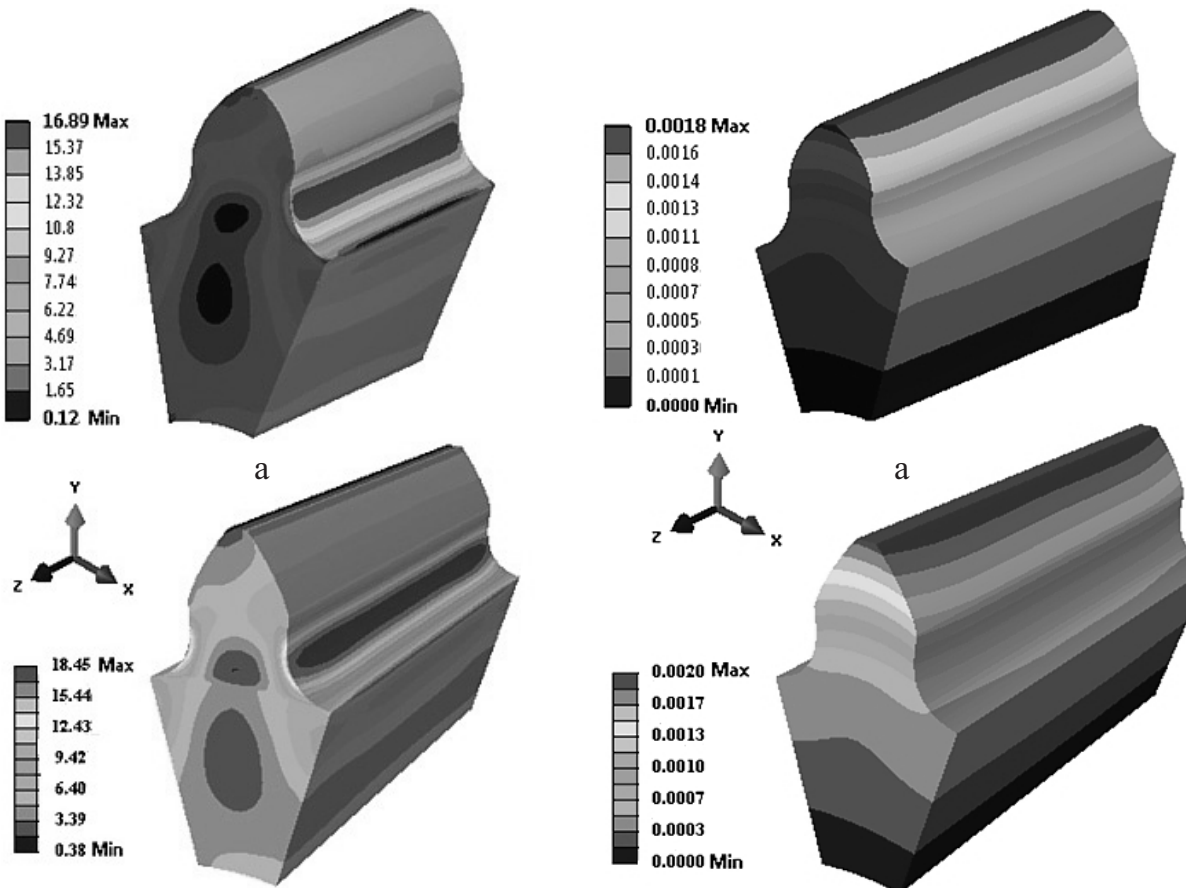

b

Fig. 8

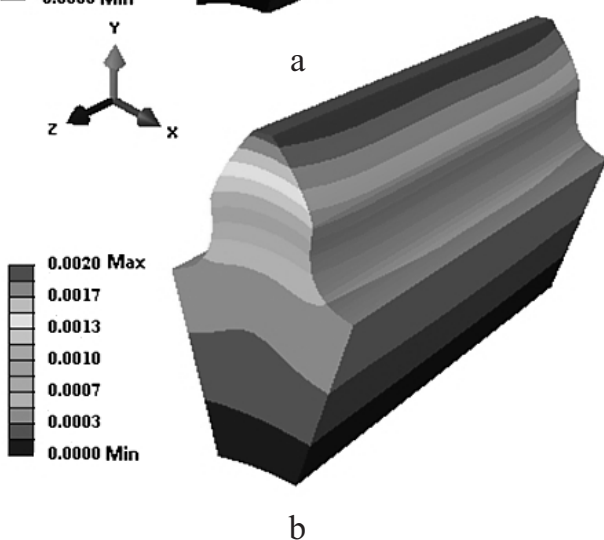

Fig. 9

Fig. 8. The Mises stress distributions in the tooth gear (MPa): (a) FEA model; (b) IGA model. Fig. 9. Total deformed shape of the tooth gear (mm): (a) FEA model; (b) IGA model.

Table 3 compares the calculated results of the tooth root stresses and deflections obtained: the bending stress results of the theoretical calculation via the Lewis equation, finite element analysis, and isogeometric analysis are in good agreement. The difference between the theoretical calculation results with those obtain via FEA and IGA are 6.47 and $2.16 \%$, respectively. The IGA approximates the theoretical calculation better than FEA.

Moreover, the IGA makes it possible to use the $h$-refinement method, in order to refine a coarse mesh of the gear geometric model, obtain additional degrees of freedom in the model, and produce the result of the required quality without changing the overall geometrical shape. This allows one to avoid the time- and cost-consuming mesh generation process in FEA, insofar as FEA requires additional meshing efforts to discretize the 3D gear tooth CAD model into fine elements for the FE analysis.

Updating the NURBS model for IGA by simulation results, in order to realize the best fillet radius to optimize the stress concentration, seems to be a quite easy task, since the geometry representation and the simulation results cover the same spline domain. However, many researchers have problems with application of the CAD-model for similar modification due to the discrepancies between CAD and FEM meshes in the FEA approach. 
Conclusions. The developed application of 3D NURBS-based high-precision model for isogeometric analysis of the stress-strain state provides not only an excellent insight into the stress distribution, but also reveals the exact position of the maximum stress value point in the gear tooth root.

The results obtained by the IGA show a good agreement with the well-known FEA method, which makes the IGA an alternative numerical method to compute spur gear stress-strain behavior under various loading conditions.

This study is mainly focused on application of the NURBS basis functions for the 3D geometric simulation of a spur gear and isogeometric analysis of stresses and deflections in the gear tooth. Further development of this tudy will include the use of other smooth parametric functions, such as T-splines [27], for the gear model geometric description and analysis. Another lucrative direction is the IGA extension to contact stress analysis.

Acknowledgments. The authors acknowledge the support by National Nature Science Foundation of China (Project Numbers 61170198 and 51305106) and the Fundamental Research Funds for the Central Universities in China.

1. D. Ristić, "Numerical model for the critical stress determination in spur gears," Sci. Tech. Rev., LVIX, No. 1, 78-86 (2009).

2. S. Sankar, M. Sundar Raj, and M. Nataraj, "Profile modification for increasing the tooth strength in spur gear using CAD," Engineering, 2, No. 9, 740-749 (2010).

3. L. Piegl and W. Tiller, The NURBS Book, 2nd ed., Springer-Verlag, BerlinHeidelberg (1995).

4. T. J. R. Hughes, J. A. Cottrell, and Y. Bazilevs, "Isogeometric analysis: CAD, finite elements, NURBS, exact geometry and mesh refinement," Comput. Methods Appl. Mech. Engrg., 194, 4135-4195 (2005).

5. Gear Technical Reference, KHK, Kohara Gear Industry Co Ltd, Japan.

6. I. Atanasovska and V. Nikolić-Stanojević, "3D spur gear FEM model for the numerical calculation of face load factor," Facta Universitatis, Ser. Mech. Autom. Contr. Robot., 6, No. 1, 131-143 (2007).

7. S. K. Tiwari and U. K. Joshi, "Stress analysis of mating involute spur gear teeth," Int. J. Eng. Res. Technol., 1, Issue 9, 2173-2180 (2012).

8. V. Karaveer, A. Mogrekar, and T. P. Reynold Joseph, "Modeling and finite element analysis of spur gear," Int. J. Current Eng. Technol., 3, No. 5, 2104-2107 (2013).

9. L. Beirão da Veiga, A. Buffa, C. Lovadina, et al., "An isogeometric method for the Reissner-Mindlin plate bending problem," Comput. Methods Appl. Mech. Engrg., 209-212, 45-53 (2012).

10. W. A. Wall, M. A. Frenzel, and C. Cyron, "Isogeometric structural shape optimization," Comput. Methods Appl. Mech. Engrg., 197, 2976-2988 (2008).

11. J. A. Cottrell, A. Reali, Y. Bazilevs, and T. J. R Hughes, "Isogeometric analysis of structural vibrations," Comput. Methods Appl. Mech. Engrg., 195, 5257-5296 (2006).

12. R. L. Taylor, "Isogeometric analysis of nearly incompressible solids," Int. J. Num. Meth. Eng., 87, No. 1-5, 273-288 (2011).

13. Y. Bazilevs, V. M. Calo, T. J. R. Hughes, and Y. Zhang, "Isogeometric fluid-structure interaction: theory, algorithms, and computations," Comput. Mech., 43, 3-37 (2008).

14. M. J. Borden, C. V. Verhoosel, M. A. Scott, et al., "A phase-field description of dynamic brittle fracture," Comput. Methods Appl. Mech. Engrg., 217-220, 77-95 (2012). 
15. C. Miehe, F. Welschinger, and M. Hofacker, "Thermodynamically consistent phasefield models of fracture: Variational principles and multi-field FE implementations," Int. J. Num. Meth. Eng., 83, No. 10, 1273-1311 (2010).

16. Y. Bazilevs, J. R. Gohean, T. J. R. Hughes, et al., "Patient-specific isogeometric fluid-structure interaction analysis of thoracic aortic blood flow due to implantation of the Jarvik 2000 left ventricular assist device," Comput. Methods Appl. Mech. Engrg., 198, 3534-3550 (2009).

17. I. Temizer, P. Wriggers, and T. J. R. Hughes, "Contact treatment in isogeometric analysis with NURBS," Comput. Methods Appl. Mech. Engrg., 200, 1100-1112 (2011).

18. L. de Lorenzis, I. Temizer, P. Wriggers, and G. Zavarise, "A large deformation frictional contact formulation using NURBS-based isogeometric analysis," Int. J. Num. Meth. Eng., 87, No. 13, 1278-1300 (2011).

19. M. E. Matzen, T. Cichosz, and M. Bischoff, "A point to segment contact formulation for isogeometric, NURBS based finite elements," Comput. Methods Appl. Mech. Engrg., 255, 27-39 (2013).

20. Q. Cheng, G. Yang, and J. Lu, "An analysis of gear based on isogeometric analysis," Vibroeng. Proc., 2, 17-22 (2013).

21. G. Marunić, "Rim stress of thin-rimmed gear," Adv. Eng., 2, 65-72 (2008).

22. W. D. Pilkey and D. F. Pilkey, Peterson's Stress Concentration Factors, 3rd ed., John Wiley \& Sons, Inc., Hoboken-New Jersey (2008), pp. 49-51.

23. L. Beirão da Veiga, D. Cho, L. F. Pavarino, and S. Scacchi, "Overlapping Schwarz methods for isogeometric analysis," SIAM J. Numer. Anal., 50, No. 3, 1394-1416 (2012).

24. C. de Falco, A. Reali, and R. Vázquez, "GeoPDEs: a research tool for Isogeometric Analysis of PDEs," Adv. Eng. Software, 42, Issue 12, 1020-1034 (2011).

25. V. P. Nguyena, S. P. A. Bordas, and T. Rabczuk, Isogeometric Analysis: An Overview and Computer Implementation Aspects, Cardiff University (2013).

26. A. Duval, H. Al-Akhras, F. Maurin, et M. Elguedj, "Implémentation d'éléments isogéométriques dans Abaqus et développement d'outils de pré- et post-traitement dédiés," en: 11e Colloque National en Calcul des Structures (CSMA 2013, 13-17 Mai 2013).

27. T. W. Sederberg, J. Zheng, A. Bakenov, and A. Nasri, "T-splines and T-NURCCs," ACM Trans. Graphics, 22, 477-484 (2003).

Received 20. 10. 2014 